\title{
ENFUSE: Engaging Fundamentals and Systems Engineering in Introductory Circuits
}

\section{Dr. Paul G. Flikkema, Northern Arizona University}

Paul G. Flikkema is Director of the Informatics \& Computing Program and a Professor of Electrical Engineering at Northern Arizona University. He is broadly interested in the properties of distributed natural and synthetic networked systems that sense and react to their environment, including decentralized and resilient systems. His current work includes research and education in energy-efficient embedded systems and networks and wireless sensor/actuator networks for monitoring and control of environmental and ecological systems. He is also active in engineering education innovation, where he has contributed to innovations in student learning of systems thinking and complex engineered systems.

Prof. Rhonda R. Franklin, University of Minnesota

Rhonda Franklin (S'84-M'96) received the B.S. degree in electrical engineering from Texas A\&M University, College Station, TX and M.S. and Ph.D. degree in electrical engineering from The University of Michigan, Ann Arbor, MI in 1990 and 1995, respectively.

She is currently a Professor with Electrical and Computer Engineering department at the University of Minnesota. Her research interests include RF/microwave passive circuit design, interconnects and integration techniques using MEMS and other advanced fabrication processes, RF fluidics, and high speed material's characterization. She has authored or co-authored over 85 professional journals and conference publications and 4 book chapters.

Dr. Franklin was the recipient of the 1998 Presidential Career Award for Scientists and Engineers by the National Science Foundation. She is an active member of the MTT-S society in the technical area of passives, packaging, integration and microwave education and is currently an Associate Editor of the IEEE Microwave Wireless Components Letters.

\section{Dr. Jeff Frolik, University of Vermont \\ Dr. Carol Haden, Magnolia Consulting, LLC}

Dr. Carol Haden is a Principal Evaluator at Magnolia Consulting, LLC, a woman-owned, small business specializing in independent research and evaluation. She has served as evaluator for STEM education projects sponsored by the National Science Foundation, the National Aeronautics and Space Administration, the William and Flora Hewlett Foundation, and the Arizona Department of Education, among others. Areas of expertise include evaluations of engineering education curricula and programs, informal education and outreach programs, STEM teacher development, and climate change education programs.

Dr. Aaron T. Ohta, University of Hawaii at Manoa

Dr. Wayne A. Shiroma, University of Hawaii at Manoa

Wayne Shiroma is Professor and Chair of Electrical Engineering at the University of Hawaii at Manoa.

Dr. Sylvia W. Thomas, University of South Florida

Dr. Tom Weller, University of South Florida

Thomas M. Weller received the B.S., M.S. and Ph.D. degrees in Electrical Engineering in 1988, 1991, and 1995, respectively, from the University of Michigan, Ann Arbor. From 1988-1990 he worked at Hughes Aircraft Company in El Segundo, CA. He joined the University of South Florida in 1995 where he is currently professor and chair in the Electrical Engineering Department. He served as the Associate Dean for Research in the College of Engineering from 2008 - 2011. He co-founded Modelithics, Inc. in 2001. Dr. Weller was a recipient of the Outstanding Young Engineer Award from the IEEE Microwave Theory and Techniques Society in 2005, the USF President's Award for Faculty Excellence in 2003, IBM Faculty Partnership Awards in 2000/2001, a National Science Foundation CAREER Award in 1999 and the IEEE MTT Society Microwave Prize in 1996. His current research interests are in the areas of 
$\mathrm{RF} /$ microwave applications of additive manufacturing, $\mathrm{RF}$ micro electromechanical systems, development and application of microwave materials, and integrated circuit and antenna design. He has 22 U.S. patents and over 250 professional journal and conference publications. 


\section{ENFUSE: Engaging Fundamentals and Systems Engineering in Introductory Circuits}

Introduction

Students' ability to connect systems-level engineering principles, and the meta-skill of systems thinking around those principles, to engineering design is important for tackling critical $21^{\text {st }}$-century challenges ${ }^{1}$. Systems-level concepts tend to be sparsely included in most engineering curricula, and, when included, tend to be relegated to upper level or Capstone Design courses. In this paper, we report on an effort to introduce students to systems thinking in Circuits, a foundational course usually taken in the freshman or sophomore years in most engineering programs of study. A unique aspect of this effort is the use of system applications related to health, environmental, and energy systems to motivate and describe one or two fundamental Circuits concepts. This is a stark departure from the historical approach of the electrical engineering curriculum where many of the fundamental concepts are presented to students first, as the foundation to understanding higher-level implementations in system applications. The effort extends the application of the team's previous efforts, which developed a blended-classroom learning environment ${ }^{2}$ for senior- and graduate-level learning of systems thinking in the context of wireless

sensor networks ${ }^{3}$, to create an approach to systems-level thinking for freshman and sophomores that is in alignment with many of the current advances in engineering education.

\section{Objectives and Overview}

This pilot study sought to answer the following questions:

How can we expose student learners to systems-centric thinking at the foundation level?

We have developed learning experiences in the context of broad technological application domains that are poised for rapid growth: Health, Energy, and Environment. The developed material exposes students to problems, objectives, and systems-level designs that carefully integrate fundamental circuit concepts with questions that stimulate systems-centric thinking. To maximize portability and adaptability to existing curriculum and courses, the content is organized into Learning Modules that can be used to supplement existing introductory circuits courses with exposure to topics that are inherently multi- and trans-disciplinary. Each module integrates circuit and systemsthinking concepts with hands-on laboratory exercises to enhance student learning and expose students to the exciting challenges awaiting them in engineering careers.

How can we provide our academic colleagues with instructional approaches that are easy and affordable to integrate into their programs, particularly during an era of shrinking budgets? 
The diversity of the investigators' five institutions - residential and commuter, large and small, with courses based on different formats (lecture, lab, and recitation)-enabled understanding of a spectrum of university-specific learning environments and cultures. Based on the diversity of needs and constraints found within our own team, we developed a methodology for integrating systems-level concepts into Circuits courses that we contend is readily transferable to other institutions.

\section{Methodology}

Each module includes a set of instructional videos and supplemental documents. The videos are designed to help motivate the module, explain important concepts, and present an experiential learning aspect.

Several hardware and software platforms were considered for the creation of the videos for the modules. Although it was ultimately decided to be agnostic to the method of content creation, some effort was made to find a recommended media creation platform. Camtasia ${ }^{\circledR}$ Studio was a top choice, as several of the authors have used this software, and it is a full-featured package that includes video creation and video editing. However, the price of implementation could be considered an obstacle to adoption by some institutions. As of this writing, a Camtasia license is $\$ 99$, and additional hardware such as a microphone may be required.

The use of apps for the Apple iPad was also explored, since all authors already had access to these tablets. Video creation apps for the iPad are considerably less expensive than Camtasia, and the iPad has cameras and microphones built-in. We piloted an app called Doceri, which is free, although it costs $\$ 4.99$ to remove a watermark from the recorded videos. However, the procedure to import Microsoft PowerPoint slides for use in the videos was a little convoluted. Thus, our group settled on another iPad app, called Explain Everything. This app is $\$ 2.99$ as of this writing, easily imports PowerPoint slides, and allows for simple video editing after recording. This app fulfilled the needs for the creation of the video content of the modules for a minimal cost (providing that an iPad is already available).

After videos for the modules were created, they were uploaded to YouTube. This enabled viewing of the videos on a wide variety of devices (computers, tablets, mobile phones), as well as the embedding of the videos on the ENFUSE website (www.uvm.edu/ enfuse).

\section{ENFUSE Module Summary}

To date, six modules have been developed and one is currently in development, to support the three systems theme areas identified in this project: Health, Environmental, and Energy. These modules in each theme area are detailed in the following. 


\section{Health Systems}

Bioelectricity - This module presents living organisms, and in particular, the human body, as electrical machines to help students link circuits and electrical systems concepts to the world around them. The instructional video that introduces the hands-on experiment first discusses the generation of bio-electrical signals and how organisms use them to control and sustain life. The main focus of the module, though, is to introduce the various ways in which bio-electrical measurements can be used to monitor the status of bio-systems. Primary attention is given to electrocardiograms, which are also the subject of the handson experiment. The video reviews resistors, capacitors and Ohm's law in the context of bioelectricity measurements in general, and then filtering ( $\mathrm{RC}$ time constants) and the block diagram of the pulse sensor electronics that are relevant to the specific ECG measurement that is made in the experiment. In this way, circuits and systems concepts are related.

During the experiment students use a commercial optical pulse sensor to measure their pulse rates and resulting waveforms are observed on an oscilloscope. Students explore analog signal processing in the form of filtering and amplification. They construct a simple RC circuit to filter the pulse waveform and investigate the effects of a varying time constant on the information content (and noise) in the signal. An operational amplifier is also used and students demonstrate the gain adjustment that is possible by selecting different resistor values. Students experience first hand the impact electrical components have on signals from biological systems.

Radiation from Wireless Devices - This module teaches systems-level thinking by giving students a pre-laboratory group exercise analyzing the radiation safety hazard from a cell phone tower along an interstate highway. This motivates the connection between AC signal frequency and power, wireless propagation, and government guidelines for health and safety, while getting students to think about their laboratory course in the context of real-world problems. They are also tasked with doing some background research, such as finding FCC data on specific absorption rates for cellular phone signals.

In this module's laboratory, students measure the level of leakage from a microwave oven, and compared this to the radiation from their own cell phones. More depth in this area is also covered by discussing antennas as resonant circuits, distributed vs. lumped circuits, frequency-selective surfaces (such as a microwave oven door), the molecular constitution of microwaveable food, and health and safety issues associated with microwave radiation.

\section{Environmental Systems}

Remote Sensing - The module asks the students to consider a remote sensing mission using BalloonSats, which are small payloads attached to a weather balloon. The BalloonSat platform has been utilized by numerous universities ${ }^{4}$ and high schools, and thus is utilized to illustrate circuit concepts in the context of environmental remote sensing. The BalloonSat platform is inexpensive, autonomous, and readily deployable, 
making it an attractive technology for rapid-response humanitarian assistance disaster relief. Students consider a mission to image an area using a camera mounted on a BalloonSat; this is applicable to scenarios such as the aftermath of a flood or other natural disaster, where imaging over a wide area can help plan recovery activities.

In this module, students build the timing circuits for activating camera shutters using a 555-timer circuit and an onboard power supply. Students learn about resistors and capacitors, parallel and series configurations, Ohm's Law, circuit analysis, and AC signals. They also explore the systems-level perspective by designing the camera system to achieve mission goals while meeting size, weight, and power constraints, as well as designing for survivability in extreme environments.

Environmental Monitoring with Sensors - This final module motivates the discussion of sensors by first presenting the opportunities enabled by the Internet of Things (IoT). Sensors, along with actuators, embedded systems, networking, etc. have enabled the rapidly developing area of IoT. Different types of sensors are introduced but the module itself focuses on three resistive based sensing devices: thermistors, photoresistors, and strain gauges. Each device is discussed in detailed and presented along with a signal conditioning circuit. These discussions leverage concepts seen early on in a Circuits course; namely, Ohm's Law, voltage division and current division. Voltage dividers are used to illustrate how to condition the response of a thermistor and photoresistor and the Wheatstone Bridge is detailed as a means to implement a strain gauge.

While detailing these three sensing devices, example applications are introduced to illustrate how these devices might be employed for smart home management or structural health monitoring. The non-linearity of, e.g., the thermistor, is discussed along with how non-linearities can be accounted for, and how they constrain a design. Both analog and digital signal conditioning are introduced, along with the corresponding costs and benefits. Other systems concepts are discussed, including the layers of abstraction required to ascertain force on a structure from the voltage measured in a strain gauge based Wheatstone bridge circuit.

This module was developed after most the others were implemented at least once at our Universities. The requisite knowledge base for this module is, as noted, among the very first of key fundamentals covered in a Circuits class. As such, this module can be readily implemented in the first weeks of the semester. In the hands-on exercise, students develop voltage dividers and a Wheatstone bridge to condition the response of a thermistor and strain gauge respectively.

\section{Energy Systems}

Power Measurement - This module is motivated by the need to improve the efficiency of electrical energy infrastructure (i.e., the grid) and the role that distributed power measurements can play in making it 'smart'. The module considers tradeoffs of invasive and direct measurement of current and non-invasive (i.e., safe) but indirect current

measurement using a split-core transformer current sensor. The module puts in context 
various aspects students may have covered in class, such voltage and current division, internal resistance requirements for voltmeters and ammeters, transformer functionality, and power factor correction. In many Circuits course offerings, AC circuits, transformers, and power are covered near the end of the semester. As such, the particular module is one that might be best offered near the end of the semester.

In the experiential component, students build their own simple current sensor that they can use to estimate the power draw of a variety of household devices. These local power measurements are put in the context of a layered view of the power system, where lower layers (appliances) interface to adjacent layers of the grid (household followed by neighborhood, etc.).

$D C$-AC Power Inversion - This module motivates the study of circuit fundamentals for $\mathrm{DC} / \mathrm{AC}$ inversion. A key objective is to introduce non-linear circuit componentsdiodes, transistors, and flip-flops - that students may not have not used, or even seen, before. In the instructional videos, students are aided in the conceptual jump to the actively-controlled transistor H-bridge using diode-based half- and full-wave rectifier circuits. In this module's experiential component, students construct a DC/AC inverter circuit, giving students a real-world example of the diversity of electronic circuits technologies and how they can be combined to create systems essential to modern life. As part of the procedure, students develop an understanding of four systems concepts: rectification, filtering, energy efficiency, and reliability. The students construct the three subsystems (clock source, control circuitry, and H-bridge) of the DC/AC inversion circuit and then interconnect them, and observe key waveforms to see the how the interplay of analog and digital electronics performs the required systems-level functions.

A strong connection to solar energy is made in an optional procedure, where students convert the DC output of a photovoltaic solar panel to AC and discover how the power output of a photovoltaic solar panel is related to its orientation to the sun. Together, the $\mathrm{DC} / \mathrm{AC}$ inverter and the solar panel provide a platform for the instructor to create numerous follow-on experiments addressing renewable energy systems. For example, using basic instrumentation and modeling techniques, students can determine the efficiency of DC/AC inversion.

Inductive Heating (in development) - This module will teach systems-level thinking by introducing students to the concept of heating using direct current (DC) and indirect current (AC) methods to transfer heat from the source to a fluid like coffee, tea, or milk. The concepts for this module introduce how heat can be developed electrically and will discuss the concept of efficiency. Power is typically usually presented in terms of generation and/or consumption. In this module, we are interested in using the power to create a heat source.

Students will learn how heat is created using resistive (DC) and inductive (AC) sources. The heat produced will be measured with a thermistor. A thermistor is a temperature dependent resistor that is able to measure the rise versus power. Students will compute the heat generated from direct heating (resistive) and indirect heating (inductive) 
measurements. They will also use a thermometer to obtain non-electrical data. To provide a tangible connection, the data from the two approaches compare and contrast the familiar measurement of temperature with the electrical measurements responsible (e.g., voltage, current, resistance or inductance, power) for producing this temperature. These concepts will provide the foundation to understanding how power is used as a heat source in a wide range of applications. Example applications include coffee heaters (now replaced with microwave heating) and induction stoves for home use, industrial applications related to heating industrial oils, and heating metal for annealing, bonding, melting, tempering or welding. This module is currently in development, with incorporation of best practices learned from the previous module development efforts.

\section{Lessons Learned}

To understand the effects of the ENFUSE content and delivery methods on increasing student engagement in and understanding of the topics, we utilized multiple evaluation methods. These included tested module feedback surveys to inform revisions to the modules as they were piloted, a student interest and understanding survey to examine impacts, and participating faculty interviews. Data collected through the feedback surveys included impacts of the modules on understanding of the topic, aspects of the modules that best supported learning of the content, aspects of the modules that students found unclear or confusing, and impacts of the modules on interest in the targeted content. The assessment process and detailed results are found in earlier work ${ }^{5}$.

Students reported an appreciation for experiential learning opportunities in the ENFUSE modules that allowed them to engage with the content in the modules. The ability to learn content within real-world contexts and situations made the concepts more accessible and relevant to them. Through real-world applications, students could understand the relationship of Circuits concepts to broader systems.

The various ENFUSE modules were developed in stages. The first module developed, Bioelectricity, was prototyped in Summer 2013 in a foundations course for non-electrical engineering majors at the University of Vermont. Based on its implementation and student feedback, the approach to the subsequent modules was modified to address student concerns. All modules (with the exception of Environmental Monitoring with Sensors) were utilized in a Circuits course at the University of South Florida in Fall 2013. After implementing these five modules, it was noted that many required concepts that were not covered in a Circuits course until well into the semester. Therefore, sequencing the modules within the curriculum is key to successful implementation: exposure to the foundational Circuits concepts and tools of a given module prior to utilizing the module led to increased interest in and understanding of the content. When the modules were used out of sequence with the foundational circuits concepts, students reported less interest and more difficulty in understanding module content.

On the basis of these findings, a new module, Environmental Monitoring with Sensors, was developed that relies on only the basic foundations of circuit theory (i.e., Ohm's law, voltage dividers, and current dividers). From an instructor's standpoint, we determined 
that the laboratory exercises created to date lacked consistency in format and were not always readily reproduced at partner institutions. Thus the experiential aspect of the Sensors module is presented in a manner similar to DYI websites (e.g., Instructables.com): a step-by-step presentation accompanied with many images to give students a clear sense of what they should be seeing in their own experiments. We believe that this approach is not only more user-friendly for the students, but can also be more readily adapted by instructors in a piece-wise manner.

\section{Conclusion}

In this work, we present a summary of a multi-university effort to create supplementary content appropriate for incorporation in first-year and sophomore Circuits courses. The effort has developed modules in the theme areas of Health Systems, Energy Systems and Environmental Systems. Each module places in core Circuits concepts in relevant systems contexts via a real application tied to one of the themes. A combination of video lectures and hands-on experiences reinforce connections to system-level concerns. The modular format also lends itself to adaptation in part $^{6}$ or adoption in full by other institutions.

This material is based upon work supported by the National Science Foundation under Grant \#DUE-1140852.

\section{Bibliography}

${ }^{1}$ The National Academy of Engineering, The Engineer of 2020: Visions of Engineering in the New Century, The National Academics Press, 2004.

2 J. Frolik, T. Weller, P. Flikkema, and C. Haden, "Implementing an Inverted Classroom Using Tablet PCs For Content Development", in The Impact of Tablet PCs and PenBased Technology on Education: Going Mainstream, Robert H. Reed and Dave A. Berque, eds., Purdue University Press, 2010.

${ }^{3}$ P.G. Flikkema, J. Frolik, C. Haden, and T. Weller, "Experiential Learning of Complex Engineered Systems in the Context of Wireless Sensor Networks", 2010 ASEE Annual Conference, Louisville, KY, June 2010.

${ }^{4}$ J. Frolik and M. Fortney, "A Low-Cost Wireless Platform for First Year Interdisciplinary Projects," IEEE Trans. Education, Vol. 49, No. 1, February 2006.

${ }^{5}$ T. Weller, C. Haden, J. Frolik, P.G. Flikkema, A. Ohta, S. Thomas, R. Franklin, and W. Shiroma, "A Systems-Centric, Foundational Experience in Circuits," 2014 ASEE Annual Conference, Indianapolis, IN, June 2014.

${ }^{6}$ P.G. Flikkema, J. Frolik, R. Franklin, C. Haden, W. Shiroma, and T. Weller, "Leveraging Multi-University Collaboration to Develop Portable and Adaptable Online Course Content", Advances in Engineering Education, Vol. 3, Issue 4, Winter 2013. 